\title{
Genetic determinants of response and survival in momelotinib- treated patients with myelofibrosis
}

Leukemia (2015) 29, 741-744; doi:10.1038/leu.2014.306

Somatic mutations (for example, CALR, ASXL1) and karyotype have been shown to independently influence survival in patients with myelofibrosis (MF). ${ }^{1,2}$ The objectives of the current study were to determine if such genetic markers also influence treatment response in MF patients receiving single agent momelotinib and whether such therapy overcomes the detrimental effect of prognostically relevant mutations in MF. Momelotinib is a Janus kinase (JAK)-1/2 inhibitor currently being evaluated in phase-3MF clinical trials (NCT01969838, NCT02101268). In an earlier phase-1/2 study $(n=166)$, the drug was shown to improve anemia (53\% response rate), reduce spleen size (39\% response rate) and alleviate constitutional symptoms ( $>50 \%$ response rate) in MF patients. ${ }^{3}$ The current study considers 100 consecutive patients, who were part of the aforementioned phase-1/2 study and received momelotinib therapy at the Mayo Clinic.

The protocol was approved by the Mayo Clinic institutional review board. Diagnosis of MF was per the 2008 World Health Organization criteria. ${ }^{4}$ Subjects for the current study were recruited at our center from those enrolled in the open-label, non-randomized study CCL09101 (NCT00935987). All subjects provided written informed consent for blood sample collection for research use. The archived samples were interrogated for specific MPN-relevant mutations and molecular data examined for correlation with treatment response and clinical outcome as per an unplanned sponsor-independent study analysis conducted in July 2014.

Study CCL09101 was conducted in two phases from November 2009 to April 2012: a single-center (Mayo Clinic) dose-escalation phase with supernumerary patient addition (Part 1), to determine the safety and tolerability of momelotinib, and to identify a therapeutic dose for the second phase, and a multicenter doseconfirmation phase (Part 2), with cohort expansion at or below the maximum tolerated dose. Momelotinib capsules were administered orally once daily with a treatment plan for continuous therapy for 36 weeks (nine $\times 28$-day cycles). Intra-patient dose escalation was permitted after completion of at least three cycles at the starting dose. Treatment beyond nine cycles was permitted on an extension study (CCL09101E; NCT01236638) if deemed beneficial to the patient and if well tolerated.

Results from Part 1 of the study (CCL09101) have been previously published. ${ }^{5}$ The published report includes details regarding study eligibility criteria and assessment of toxicity and treatment response. Specifically, responses were measured every 4 weeks per the 2006 IWG-MRT criteria; $^{6}$ clinical data including palpable spleen and liver size and details regarding packed red blood cell (RBC) transfusions were recorded at every study visit. Interim data for the overall study (Parts 1 and 2) have been previously reported in abstract form. ${ }^{3}$ Previously published methods were used for mutation analyses. ${ }^{7}$

All statistical analyses considered clinical and laboratory parameters obtained at the time of entry to study CCL09101. Differences in the distribution of continuous variables between categories were analyzed by either Mann-Whitney or
Kruskal-Wallis test. Patient groups with nominal variables were compared by chi-square test. Survival analysis was considered from the date of study entry to the date of death (uncensored) or last contact (censored). Leukemia-free survival calculations considered leukemic transformation as the uncensored variable. Survival curves were prepared by the Kaplan-Meier method and compared by the log-rank test. Cox proportional hazard regression model was used for multivariate analysis of survival. Logistic regression methods were used for multivariable analysis of response predictors. $P$ values $<0.05$ were considered significant. The Stat View (SAS Institute, Cary, NC, USA) statistical package was used for all calculations.

One hundred patients with intermediate- or high-risk MF were enrolled in study CCL09101 at our site (60 and 40 patients in Parts 1 and 2, respectively); 64 patients had PMF, 22 post-polycythemia vera MF and 14 post-essential thrombocythemia MF. Detailed demographic, clinical and laboratory characteristics of this cohort are presented in Table 1. Twenty-one patients $(21 \%)$ had been previously treated with an alternative JAK inhibitor; other treatments included thalidomide in 11 patients, pomalidomide in 18 patients and lenalidomide in 11 patients. The momelotinib starting dose was $100 \mathrm{mg} /$ day $(n=3), 150 \mathrm{mg} /$ day $(n=21), 150 \mathrm{mg}$ twice daily $(n=20), 200 \mathrm{mg} /$ day $(n=3), 300 \mathrm{mg} /$ day $(n=47)$ and $400 \mathrm{mg} /$ day $(n=6)$. The maximum momelotinib dose achieved was $150 \mathrm{mg} /$ day $(n=6), 150 \mathrm{mg}$ twice daily $(n=20), 300 \mathrm{mg} /$ day $(n=68)$ and $400 \mathrm{mg} /$ day $(n=6)$. Seventy-one patients $(71 \%)$ required dose reduction at some point during treatment. After a median (range) follow-up of 36 months (1-52), 83 patients (83\%) had discontinued momelotinib treatment. During the follow-up period, 57 (57\%) deaths and $12(12 \%)$ leukemic transformations were recorded.

Eighty-seven patients were evaluable for spleen response and in addition four splenectomized patients were evaluable for liver response; the median (range) palpable spleen size at baseline was $19 \mathrm{~cm}$ (6-32). Sixty-eight patients (68\%) were evaluable for anemia response; of these 49 patients (49\%) were RBC transfusiondependent at study enrollment. Thirty-eight patients achieved a spleen response and one of four splenectomized patients a liver response, for an overall response rate of $43 \%$. Thirty patients (44\%) achieved an anemia response, including 25 who were RBC transfusion-dependent at baseline.

Spleen response was correlated with JAK2/MPL/CALR and CALR/ ASXL1 mutational status, with the greatest benefit conferred by CALR mutated status (Table 1), and, additionally, by smaller palpable spleen $(57 \%$ vs $\geqslant 30 \%$ response rate for less or more than the median spleen size; $P=0.02$ ) and constitutional symptoms (absent $57 \%$ vs present $34 \% ; P=0.049$ ). On multivariable analysis, only CALR mutated (hazard ratio $(\mathrm{HR})=0.2,95 \%$ confidence interval $(\mathrm{Cl})=0.04-0.6)$ and $A S X L 1$ unmutated $(\mathrm{HR}=0.3,95 \%$ $\mathrm{Cl}=0.1-0.8)$ status were independently associated with spleen response. Anemia response was not correlated with mutational status (Table 1), baseline karyotype or other identifiable clinical parameter. However, anemia response in RBC transfusiondependent patients $(n=49)$ was correlated with DIPSS-plus status (intermediate-2 $100 \%$ vs high $41 \% ; P=0.004$ ), baseline karyotype 


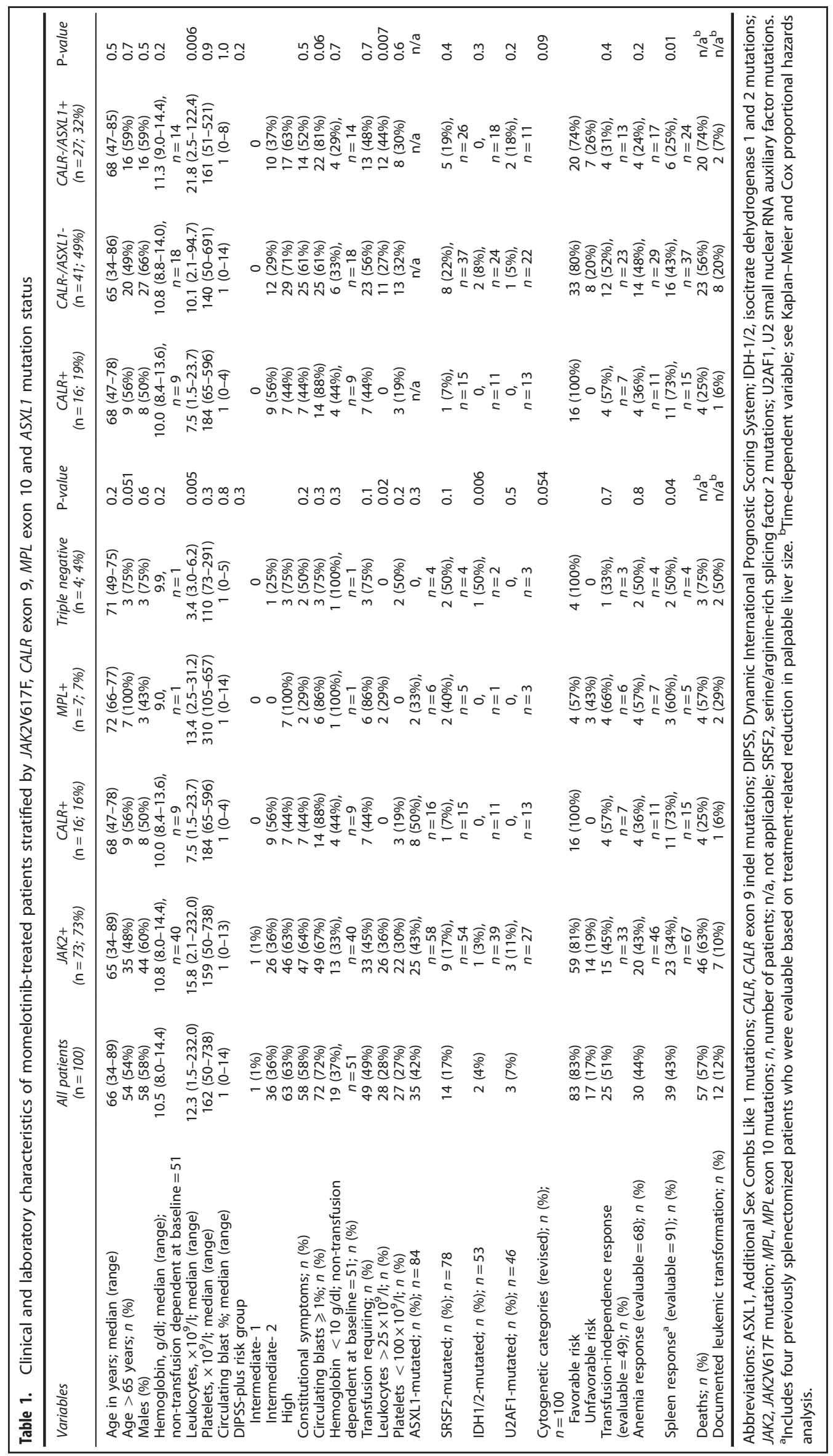


Survival in 84 momelotinib-treated patients with myelofibrosis stratified by their CALR/ASXL1 mutational status

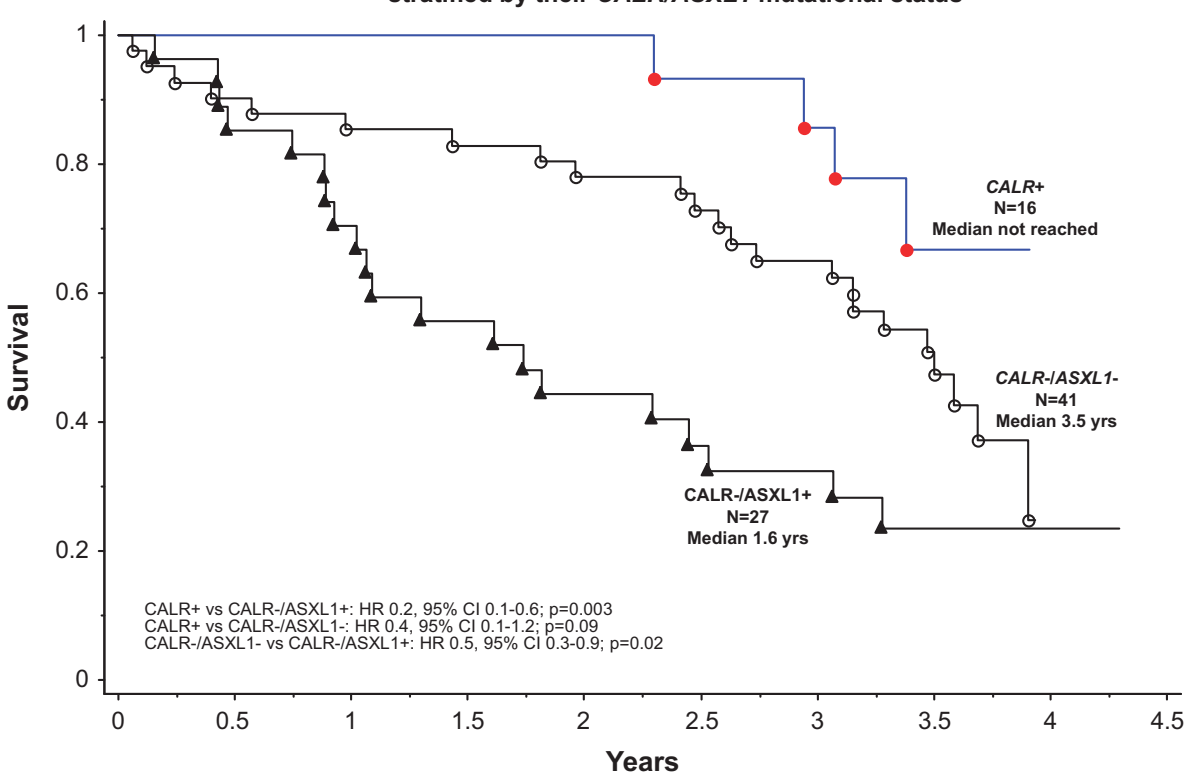

Figure 1. Kaplan-Meier survival curves of 84 myelofibrosis patients treated with single agent momelotinib stratified by CALR/ASXL1 mutation status. Survival was calculated from the time of study entry.

(normal $70 \%$ vs abnormal $38 \% ; P=0.04$ ) and platelet count $\left(\geqslant 100 \times 10^{9} /\right.$ / $59 \%$ vs $\left.<100 \times 10^{9} / / 25 \% ; P=0.05\right)$.

Absence of CALR ( $\mathrm{HR}=3.4,95 \% \mathrm{Cl}=1.2-9.4)$, and presence of ASXL1 $(\mathrm{HR}=2.0,95 \% \mathrm{Cl}=1.1-3.5)$ or SRSF2 $(\mathrm{HR}=2.6,95 \% \mathrm{Cl}=1.3-$ 5.0) mutations was associated with inferior survival, independent of age $>65$ years or DIPSS-plus risk status. Patients who were CALR+ $(\mathrm{HR}=0.2,95 \% \mathrm{Cl}=0.07-0.6)$ or CALR-/ASXL1 - $(\mathrm{HR}=0.5$, $95 \% \mathrm{Cl}=0.3-0.9)$ lived longer than those with CALR-/ASXL1+ mutational status (Figure 1). This relationship was independent of SRSF2 mutation status $(\mathrm{HR}=0.2,95 \% \mathrm{Cl}=0.1-0.7$ and $\mathrm{HR}=0.5$, $95 \% \mathrm{Cl}=0.3-0.96$, respectively), DIPSS-plus risk status $(\mathrm{HR}=0.2$, $95 \% \mathrm{Cl}=0.1-0.7$ and $\mathrm{HR}=0.5,95 \% \mathrm{Cl}=0.3-0.9$, respectively) or momelotinib dose $(\mathrm{HR}=0.3,95 \% \mathrm{Cl}=0.1-0.9$ and $\mathrm{HR}=0.5,95 \%$ $\mathrm{Cl}=0.3-0.99$, respectively). CALR/ASXL1 mutational status did not correlate with leukemia-free survival, which was, however, inferior in 'triple-negative (JAK2-/CALR-/MPL - )' vs CALR+ patients (HR= $13.4,95 \% \mathrm{Cl}=1.2-153)$.

Our observations on survival are consistent with recent reports in the general population of patients with MF. ${ }^{1,7,8}$ In other words, momelotinib treatment was unable to overcome the negative prognostic impact of mutational status in MF. This was also the case with ruxolitinib, another JAK-1/2 inhibitor. ${ }^{9}$ However, in contrast to our observation with momelotinib, ruxolitinib-induced spleen response in the latter study, which predated the discovery of CALR mutations, was reported to be independent of mutational status. ${ }^{9}$ Regardless, the data from the current study require confirmation from the ongoing larger phase-3 studies of momelotinib therapy in MF. Similarly, it remains to be seen whether our observations are generalizable to other JAK inhibitors. Finally, the basis for the discordance in terms of spleen versus anemia response vis-à-vis CALR mutation status in the current study suggests distinct pathogenetic mechanisms of response.

\section{CONFLICT OF INTEREST}

AT was Principal Investigator for studies CCL09101 and CCL09101E for the Mayo Clinic (Rochester) site. AP and AT are/have served as Principal Investigator for other JAK inhibitor clinical trials for myelofibrosis treatment. The remaining authors declare no conflict of interest.

\section{ACKNOWLEDGEMENTS}

The sponsors (Cytopia, YM Biosciences, Gilead) funded the aforementioned clinical trials and provided the study drug, but were not involved in the current substudy or in manuscript preparation.

\section{AUTHOR CONTRIBUTIONS}

AP and AT designed the study, analyzed the data and wrote the first draft of the manuscript; RAA abstracted the clinical data; CF and TTL performed the molecular analysis; KHB, AA-K, WJH and MRL contributed patients/patient samples to the study; CAH reviewed bone marrow histology data; and RPK reviewed the cytogenetic data. All authors reviewed, provided input and approved the final draft of the manuscript.

A Pardanani ${ }^{1}$, RA Abdelrahman ${ }^{1}$, C Finke ${ }^{1}$, TT Lasho ${ }^{1}$, KH Begna ${ }^{1}$, A Al-Kali ${ }^{1}$, WJ Hogan ${ }^{1}$, MR Litzow ${ }^{1}$, CA Hanson ${ }^{2}$, RP Ketterling ${ }^{3}$ and A Tefferi ${ }^{1}$

${ }^{1}$ Division of Hematology and Department of Medicine, Department of Laboratory Medicine, Mayo Clinic, Rochester, MN, USA;

${ }^{2}$ Division of Hematopathology, Department of Laboratory Medicine, Mayo Clinic, Rochester, MN, USA and

${ }^{3}$ Division of Cytogenetics, Department of Laboratory Medicine, Mayo Clinic, Rochester, MN, USA E-mail: tefferi.ayalew@mayo.edu

\section{REFERENCES}

1 Tefferi A, Guglielmelli P, Larson DR, Finke C, Wassie EA, Pieri L et al. Long-term survival and blast transformation in molecularly-annotated essential thrombocythemia, polycythemia vera and myelofibrosis. Blood 2014; 124: 2507-2513.

2 Vannucchi AM, Lasho TL, Guglielmelli P, Biamonte F, Pardanani A, Pereira A et al. Mutations and prognosis in primary myelofibrosis. Leukemia 2013; 27: 1861-1869.

3 Pardanani A, Gotlib J, Gupta V, Roberts A, Wadleigh M, Sirhan S et al. Update on the long-term efficacy and safety of momelotinib, a JAK1 and JAK2 inhibitor, for the treatment of myelofibrosis. Blood 2013; 122.

4 Vardiman JW, Thiele J, Arber DA, Brunning RD, Borowitz MJ, Porwit A et al. The 2008 revision of the World Health Organization (WHO) classification of myeloid neoplasms and acute leukemia: rationale and important changes. Blood 2009; 114: 937-951.

5 Pardanani A, Laborde RR, Lasho TL, Finke C, Begna K, Al-Kali A et al. Safety and efficacy of CYT387, a JAK1 and JAK2 inhibitor, in myelofibrosis. Leukemia 2013; 27: 1322-1327. 
6 Tefferi A, Barosi G, Mesa RA, Cervantes F, Deeg HJ, Reilly JT et al. International Working Group (IWG) consensus criteria for treatment response in myelofibrosis with myeloid metaplasia, for the IWG for Myelofibrosis Research and Treatment (IWG-MRT). Blood 2006; 108: 1497-1503.

7 Tefferi A, Guglielmelli P, Lasho TL, Rotunno G, Finke C, Mannarelli C et al. CALR and ASXL1 mutations-based molecular prognostication in primary myelofibrosis: an international study of 570 patients. Leukemia 2014; 28: 1494-1500.

8 Guglielmelli P, Lasho TL, Rotunno G, Score J, Mannarelli C, Pancrazzi A et al. The number of prognostically detrimental mutations and prognosis in primary myelofibrosis: an international study of 797 patients. Leukemia 2014; 28 1804-1810.
9 Guglielmelli P, Biamonte F, Rotunno G, Artusi V, Artuso L, Bernardis I et al. Impact of mutational status on outcomes in myelofibrosis patients treated with ruxolitinib in the COMFORT-II study. Blood 2014; 123: 2157-2160.

This work is licensed under a Creative Commons AttributionNonCommercial-NoDerivs 4.0 International License. The images or other third party material in this article are included in the article's Creative Commons license, unless indicated otherwise in the credit line; if the material is not included under the Creative Commons license, users will need to obtain permission from the license holder to reproduce the material. To view a copy of this license, visit http:// creativecommons.org/licenses/by-nc-nd/4.0/

\section{Phenotypic heterogeneity in $I G H V$-mutated CLL patients has prognostic impact and identifies a subset with increased sensitivity to BTK and PI3K $\delta$ inhibition}

Leukemia (2015) 29, 744-747; doi:10.1038/leu.2014.308

The majority of chronic lymphocytic leukemia (CLL) patients are diagnosed with early-stage disease but the currently used prognostic tools appear to be less informative in this group of patients. ${ }^{1}$ This is especially problematic for patients with mutated immunoglobulin genes (M-CLL) as they have a more diverse clinical course when compared with patients with unmutated immunoglobulin genes (U-CLL). ${ }^{1-4}$ Given the emergence of promising targeted, less toxic, therapeutics in $\mathrm{CLL}_{\text {, }}^{5,6}$ there is an increased need to identify patients who might benefit from early treatment with these new agents.

Chemokine receptors expressed on CLL cells are thought to regulate the trafficking of the leukemic cells between blood and lymphoid tissues. ${ }^{5}$ Logically, the tendency of CLL cells to return to tissue sites where they are cytoprotected and are driven to proliferate contributes to clinical aggressiveness. It is therefore possible that these receptors represent promising prognostic markers and potentially useful therapeutic targets. We previously reported that one such chemokine receptor, CXCR4, is associated with poor clinical outcome in an unselected cohort of CLL patients. ${ }^{7}$ Here we specifically focused our attention on M-CLL samples to ascertain the impact of CXCR4 expression in this clinically heterogeneous subset. The cohort was made up of 60 $\mathrm{M}-\mathrm{CLL}$ patients from the original study and 64 additional M-CLL patients. The patient characteristics are given in Supplementary Table 1. CXCR4 expression was determined using the three-color flow cytometric assay described previously. ${ }^{7}$ We used the median expression as a binary classifier based on receiver operating characteristic analysis. Of the $124 \mathrm{M}-\mathrm{CLL}$ samples analyzed, 50 (40.4\%) were classified as CXCR4 ${ }^{\text {hi }}$ and 74 (59.6\%) as CXCR4 ${ }^{\text {lo; }}$; M-CLL patients showed much more heterogeneity in CXCR4 expression than U-CLL (Figure 1a). Importantly, elevated CXCR4 expression in M-CLL was a strong determinant of reduced overall survival (Figure $1 \mathrm{~b}$; hazard ratio $(H R)=3.5$ ). As $M-C L L$ patients utilizing IGHV3-21 genes have been shown to have an inferior clinical outcome, 8,9 we asked whether the CXCR4 expression was significantly different in the IGHV3-21 subset. We found no significant increase in CXCR4 expression in IGHV3-21 samples when compared with samples utilizing other IGHV gene segments (Supplementary Figure 1; $P=0.92$ ). Similarly, we found no association between CXCR4 expression and the high-risk cytogenetic risk groups (Supplementary Figure 1; $P=0.41$ ).

We recently showed a strong association between CXCR4 expression and CD49d in an unselected cohort of U-CLL and M-CLL patients. ${ }^{7}$ We therefore examined the expression of CD49d in this M-CLL subset using the same flow cytometric methodology and determined its prognostic relevance. In concordance with our CXCR4 data, there was heterogeneous expression of CD49d with $60(47.4 \%)$ CD49d $d^{\text {hi }}$ and $64(52.6 \%)$ CD49 $d^{\text {lo. }}$. Again this heterogeneity was in marked contrast to $\mathrm{U}-\mathrm{CLL}$ cases (Figure 1c). Furthermore, CD49d $\mathrm{d}^{\text {hi }}$ patients ( $>30 \%$ positive) had a significantly worse clinical outcome than CD49d ${ }^{\text {lo }}$ patients (Figure $1 \mathrm{~d}$; $H R=3.4$ ) reinforcing the credentials of CD49d as a prognostic marker in the M-CLL subset. ${ }^{10}$ When assessed as continuous variables, CXCR4 and CD49d were strongly correlated (Figure 1e; $P<0.0001$ ). Using categorical cutoffs to define the cohort, the majority of M-CLL cases showed concordant expression for CXCR4 and CD49d: CXCR4 ${ }^{\text {hi }} /{\text { CD } 49 d^{\text {hi }}}$ or CXCR4 $4^{\mathrm{lo}} / \mathrm{CD} 49 \mathrm{~d}^{\mathrm{lo}}$. However, $27 \%$ of the subset was discordant for these markers (Supplementary Figure 2). The Kaplan-Meier curve for the discordant cases bisected the CXCR4 ${ }^{\text {hi }} /$ CD $49 \mathrm{~d}^{\text {hi }}$ and CXCR4 ${ }^{\text {lo }} /$ CD $49 \mathrm{~d}^{\mathrm{lo}}$ curves highlighting the prognostic importance of both CXCR4 and CD49d and suggesting that the combined assessment of CXCR4 and CD49d has clinical utility. This was supported by the observation that the combination of CXCR4 and CD49d was a more powerful prognostic tool than either marker alone for the concordant cases (Figure $1 \mathrm{f} ; \mathrm{HR}=5.2$ ). It is worthy of note that CXCR4 $4^{\text {hi }} / C D 49 d^{\text {hi }}$ M-CLL cases had a similar clinical outcome when compared with U-CLL cases (Supplementary Figure 3).

We went on to establish the functional significance of CXCR4 and CD49d expression in M-CLL. CXCR4 ${ }^{\text {hi }} / C D 49 d^{\text {hi }}$ samples showed significantly increased migration in response to CXCL12 in transwell experiments (Figure 2a; $P=0.012$ ), which could be inhibited by both plerixafor (CXCR4 antagonist) and natalizumab (anti-CD49d antibody) even in the presence of CXCL12 (Figure 2b). We observed a significant reduction in CXCR4 expression when CLL cells were incubated with CXCL12 presumably owing to the 